\title{
Antibacterial treatment of LDPE with halogen derivatives via cold plasma
}

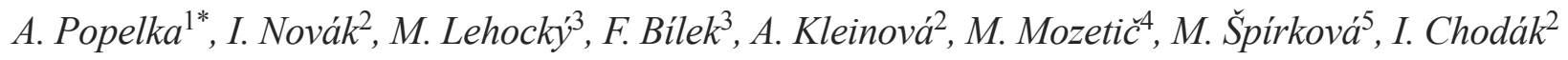 \\ ${ }^{1}$ Center for Advanced Materials, Qatar University, P.O. Box 2713, Doha, Qatar \\ ${ }^{2}$ Polymer Institute, Slovak Academy of Sciences, Dúbravská cesta 9, 84541 Bratislava 45, Slovakia \\ ${ }^{3}$ Centre of Polymer Systems, University Institute, Tomas Bata University in Zlín, Nad Ovčírnou 3685, 76001 Zlín, Czech \\ Republic \\ ${ }^{4}$ Department of Surface Engineering, Jožef Stefan Institute, Jamova cesta 39, SI-1000 Ljubljana, Slovenia \\ ${ }^{5}$ Institute of Macromolecular Chemistry, Academy of Sciences of the Czech Republic, Heyrovsky Sq. 2, 16006 Prague, \\ Czech Republic
}

Received 25 August 2014; accepted in revised form 14 November 2014

\begin{abstract}
The factor limiting the application of low-density polyethylene (LDPE) in healthcare is its high susceptibility to bacterial growth. For this reason, we here investigated antibacterial treatments of LDPE foils using appropriate antibacterial agents. Benzalkonium chloride and bronopol were selected because of their satisfactory antibacterial effect, which has been confirmed by their application in the medical and cosmetic industries. The aforementioned substances were immobilized by a multistep approach via the grafting of polyacrylic acid (PAA) brushes onto LDPE surfaces pre-treated with low-temperature plasma. Measurements of the surface energy, peel strength of the adhesive joints, X-ray photoelectron spectroscopy (XPS), Fourier-transform infrared spectroscopy with attenuated total reflectance (FTIR-ATR), and atomic force microscopy (AFM) were used to investigate the surface and adhesive properties of the antibacterial-treated LDPE. Moreover, the antibacterial effect was determined via measurements of the inhibition zone of the Staphylococcus aureus (S. aureus) bacterial strain. The antibacterial activity of benzalkonium chloride was observed to be more pronounced than that of bronopol. Inhibition-zone measurements of Escherichia coli (E. coli) were also conducted, but an antibacterial effect was not observed.
\end{abstract}

Keywords: adhesion, benzalkonium chloride, bronopol, plasma treatment, antibacterial activity

\section{Introduction}

Polyethylene (PE) has found uses in various biomedical applications [1], such as the production of catheters [2]. However, PE often leads to unfavorable infections that represent serious clinical complications [3]. These infections can result in implant failure, which can lead to numerous health problems [4]. To overcome this disadvantage, an antiinfection modification of PE is frequently applied. Anti-infective properties of polymers are achieved by the incorporation of an anti-infection agent into the polymer bulk or by copolymerization of an antiinfection agent with a monomer. Recently, an anti- bacterial surface treatment was demonstrated to be the preferred choice because it exhibited several advantages: it did not influence the bulk properties of the polymer, antibacterial agents were not released from the polymer volume, and the procedure was relatively simple and effective [5].

In this study, a multistep approach consisting of plasma surface treatment, radical graft polymerization $[6,7]$ of acrylic acid (AA) and the immobilization of antibacterial agents, was used (Figure 1) [8]. Surface treatment with low-temperature plasma plays a pronounced role in the surface treatment of polymers without influencing their volume properties

\footnotetext{
*Corresponding author, e-mail: anton.popelka@qu.edu.qa

(C) BME-PT
} 

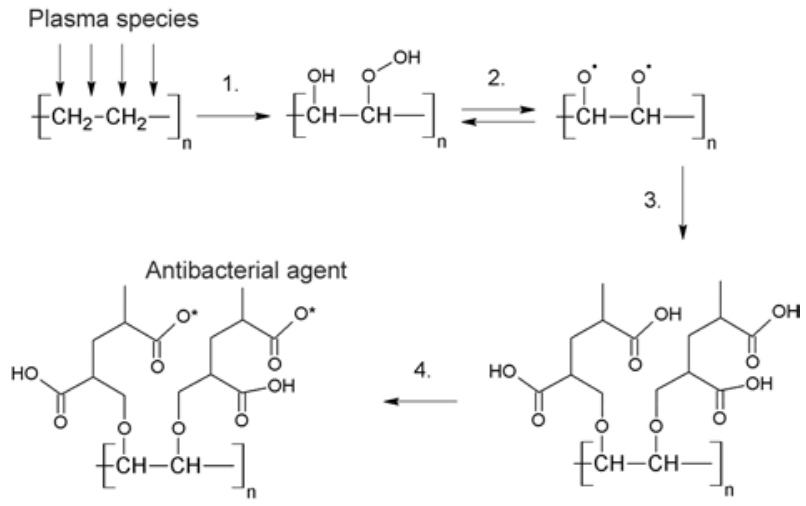

Figure 1. Multistep approach of the antibacterial treatment: 1. plasma treatment; 2. radicals generation; 3. AA radical graft polymerization; and 4 . immobilization of the antibacterial agents

$[9,10]$. Moreover, the use of low-temperature plasma is an environmental friendly, clean and dry process [11]. Low-temperature plasma facilities are often used in the electronic, aerospace, automotive, textile and biomedical industries $[12,13]$. The products of the low-temperature plasma discharge, such as electrons, ions and excited atoms, are capable of interacting with the solid polymer surface. These interactions can lead to the functionalization (i.e., the creation of functional groups), etching (i.e., ablation), polymerization, or cross-linking processes [14]. Diffuse coplanar surface barrier discharge (DCSBD) plasma generators are used by facilities to generate the high surface power density of plasma discharges. The main advantage of this plasma system is its ability to operate at atmospheric pressure (i.e., it is suitable for continuous processes) [15]. The lifetime of electrodes is prolonged through the use of electrodes embedded in $\mathrm{Al}_{2} \mathrm{O}_{3}$ and connected to a cooling system [16]. Moreover, the high surface density of plasma generates visually macroscopically homogeneous low-temperature plasma discharges [17].

Low-temperature plasma can also initiate a radical graft polymerization of acrylic acid (AA) on the polymer surface [18]. The brushes of the resulting polyacrylic acid (PAA) represent an effective interfacial layer for antibacterial immobilization [19]. Benzalkonium chloride (Figure 2a) is known to be a safe, nontoxic biocide and is currently used in human pharmaceuticals [20]. Bronopol (Figure 2b) is another efficient antibacterial agent with a low clinical toxicity and as an effective protecting agent is used in various applications (e.g., water protection, cosmetics) [21]. These antibacterial agents are capable of inhibiting the proliferation of microorganisms on

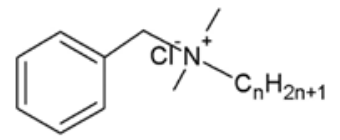

a)

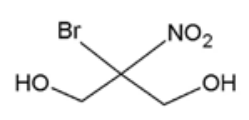

b)
Figure 2. Scheme of: a) benzalkonium chloride and b) bronopol

the polymer surface [22]. The antibacterial activity of treated LDPE was investigated as a tool for eliminating the bacterial strains Staphylococcus aureus (S. aureus)-a gram-positive coccal bacterial strain that can lead to, for example, urinary tract infections and Escherichia coli (E. coli), which is a gramnegative rod-shaped bacteria that causes, for example, intestinal disease [23].

\section{Experimental}

\subsection{Materials}

Branched low-density polyethylene LDPE foils BRALEN FB $2-17\left(T_{\mathrm{m}}=114^{\circ} \mathrm{C}, T_{\mathrm{g}}=-110^{\circ} \mathrm{C}, M_{\mathrm{w}}=\right.$ 260000 and PDI $=22.5$ ) with a thickness of $20 \mu \mathrm{m}$ were used in this study. The foils were supplied by Slovnaft-MOL (Slovakia) and contained no additives. These LDPE foils comply with the Food Contact Regulations and are approved for food contact; in addition, the grade is appropriate for use in pharmaceutical packaging products. Acrylic acid (AA, 99.0\%, anhydrous, Acros Organics, Belgium) was used for the radical graft polymerization. Sodium metabisulfite (99.0\%, Reagent plus, Merck, USA) was used as an inhibitor of acrylic acid homopolymerization. $N$-(3-Dimethylaminopropyl)- $N$ '-ethylcarbodiimide hydrochloride (EDAC, 98.0\%, Fluka, USA) was used as a carboxyl-group activator for enabling immobilization of antibacterial agents. Benzalkonium chloride (benzyl-dimethyl-tridecyl-azanium chloride, Sigma-Aldrich, Denmark) and bronopol (2-bromo-2-nitropropane-1,3-diol, Fluka, Germany) were used as antibacterial agents. Ultrapure water (produced by a Heal Force NW ULTRA pure water system) was used in the experiments. Diiodomethane (99.0\%, Reagent plus), formamide (99.5\%, molecular biology grade), and glycerol ( $99 \%$, for molecular biology), which were supplied by Sigma-Aldrich (USA), were used as testing liquids for the surface wettability measurements.

\subsection{Plasma treatment}

The DCSBD plasma system developed by Černák et al. [15] was used to activate the LDPE foils. The 
surface treatment was performed under dynamic conditions (i.e., the samples were in motion above the electrodes) at atmospheric pressure; the surface power density was $1 \mathrm{~W} / \mathrm{cm}^{2}$, the duration of the plasma treatment was $15 \mathrm{~s}$, and air atmosphere was used as a working gas. Both sides of all samples were treated. A schematic of the DCSBD plasma equipment is shown in Figure 3. Two parallel banded systems consisting of several electrodes made of $\mathrm{Ag}$ paste $(1 \mathrm{~mm}$ wide, $50 \mu \mathrm{m}$ thick, and $0.5 \mathrm{~mm}$ spacing between the strips) were used to generate the low-temperature plasma discharges in an effective manner. These electrodes were embedded in $96 \% \mathrm{Al}_{2} \mathrm{O}_{3}$, which protects the electrodes from direct contact with the plasma discharges and prolongs their durability. Moreover, the plasma panel was connected to a cooling system to prevent its overheating. A high-frequency sinusoidal voltage $\left(\approx 15 \mathrm{kHz}, U_{\mathrm{m}} \approx 10 \mathrm{kV}\right)$ was used to provide power to the electrodes led to the generation of
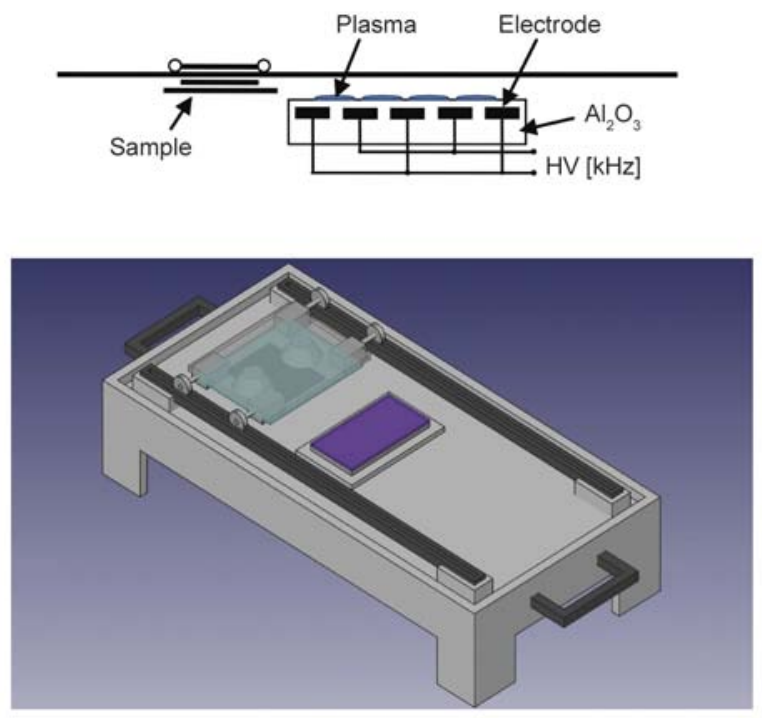

Figure 3. Schematic of the DCSBD plasma system macroscopically homogenous plasma and resulted in a uniform surface treatment.

\subsection{PAA grafting}

Grafting of PAA onto the surface of the LDPE foil was performed immediately after plasma treatment by immersing the activated foil into a $10 \mathrm{vol} \%$ aqueous solution of AA for $24 \mathrm{~h}$ at $30^{\circ} \mathrm{C}$. The solution also contained $0.1 \mathrm{wt} \%$ sodium metabisulfite as an efficient inhibitor of AA homopolymerization. The AA polymerization reaction led to the formation of PAA brushes, such was seen from AFM measurement and rendered it suitable for the effective immobilization of antibacterial agents. Finally, the prepared samples were thoroughly washed in distilled water for $5 \mathrm{~min}$ at $30^{\circ} \mathrm{C}$ to remove weakly bound or unreacted AA. The pre-treated samples were used for the immobilization of the antibacterial agents.

\subsection{Benzalkonium chloride and bronopol immobilization}

The PAA grafted substrates were subsequently immersed in a $0.1 \%(\mathrm{w} / \mathrm{v})$ aqueous solution of EDAC at $4{ }^{\circ} \mathrm{C}$ for 6 hours to activate the carboxyl groups, as shown in Figure 4. O-Acylisourea moieties are formed by this activation, and the PAA brushes are subsequently able to react with certain reducing agents. Two procedures were used: pretreated LDPE samples were immersed in a benzalkonium chloride $2 \%(\mathrm{w} / \mathrm{v})$ solution in distilled water for 24 hours at $30^{\circ} \mathrm{C}$, and, alternatively, pretreated LDPE samples were immersed in a bronopol $2 \%(\mathrm{w} / \mathrm{v})$ solution in absolute ethanol for 24 hours at $30^{\circ} \mathrm{C}$. Finally, samples with an immobilized antibacterial agent were washed in water and dried for $24 \mathrm{~h}$ at room temperature.

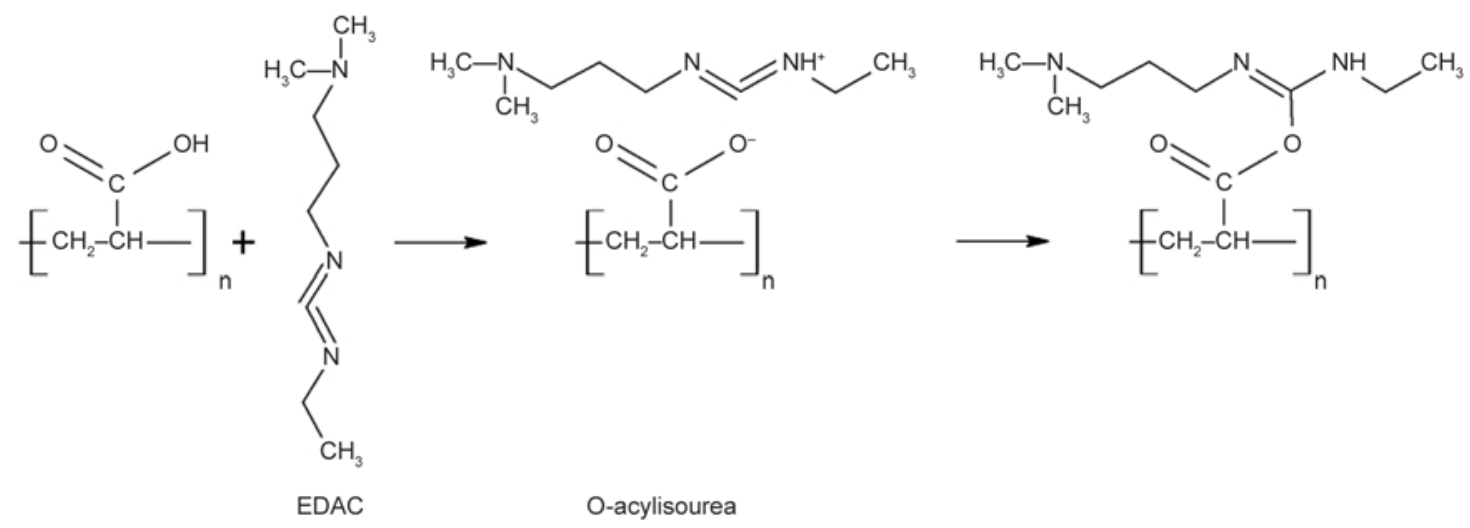

Figure 4. Carboxyl-group activation by EDAC 


\subsection{Surface wettability investigation}

The surface wettability of the samples was investigated via sessile drop contact angle measurements. A surface energy evaluation system (SEE system, Advex-Instruments, Czech Republic) was used in this study. The CCD camera resolution was set to $1280 \times 960$ pixels to ensure that high-quality images of the drops were recorded. The contact angle refers to the angle between the solid/liquid interface and the liquid/vapor interface. Ultra-pure water, ethylene glycol, glycerol, formamide, and diiodomethane were used as test liquids. The contact angle was recorded after $3 \mathrm{~s}$ to allow thermodynamic equilibrium to be established between the solid, liquid, and gas phases. Average values of contact angles were used to calculate the surface free energy $\left(\gamma^{\text {tot }}\right)$, and its polar $\left(\gamma^{\mathrm{p}}\right)$ and dispersive $\left(\gamma^{\mathrm{d}}\right)$ components were evaluated using the Owens-Wendt-Rabel-Kaelble regression model [24].

Moreover, changes in the graft yield of LDPE samples obtained by gravimetric measurements are also included. The graft yield $(G Y)$ was calculated by Equation (1):

$$
G Y=\frac{W_{2}-W_{1}}{W_{1}} \cdot 100[\%]
$$

where $W_{1}$ and $W_{2}$ are the weights of the samples before and after the surface treatment [25].

\subsection{Adhesive properties assessment}

The adhesion properties between two materials are characterized by peel strength (force per unit width). The adhesive joint created from LDPE samples and poly(2-ethyhexyl acrylate) deposited on $15 \mathrm{~mm}$ polypropylene foil were used for the peel-strength measurements. Steel roller $(3 \mathrm{~kg})$ was used for the preparation of adhesive joint with constant distribution of adhesive forces. We performed these measurements by conducting $90^{\circ}$ peel tests at a peel rate $10 \mathrm{~mm} / \mathrm{min}$ using a universal INSTRON 4301 (UK) dynamometer equipped with $100 \mathrm{~N}$ load cell. The end parts of the LDPE samples were firmly fixed in clamps in a circular arrangement to achieve an even distribution of tension across the entire width. PP foil with an adhesive as a second part of the adhesive joint was fixed in the clamp connected to the load cell.

\subsection{Surface morphology analysis}

Atomic force microscopy (AFM) investigations of the surface topography were performed on a com- mercial atomic force microscope (Dimension Icon, Bruker) equipped with an SSS-NCL probe, Super Sharp SiliconTM-SPM-Sensor (NanoSensorsTM Switzerland; spring constant $35 \mathrm{~N} / \mathrm{m}$, resonant frequency: $170 \mathrm{kHz}$ ). The areas for individual AFM measurements were carefully chosen after previous screening of the surface regularity. Measurements were performed under ambient conditions and in tapping mode. The scans covered the sizes from $0.5 \times 0.5$ to $10 \times 10 \mu \mathrm{m}^{2}$. Characteristic AFM images for each sample (area $10 \times 10 \mu \mathrm{m}^{2}$ ) are represented in this paper.

\subsection{Surface chemistry investigation}

Fourier-transform infrared spectroscopy with attenuated total reflectance (FTIR-ATR) was used to determine the chemical composition of LDPE samples. The individual measurements were performed with a FTIR NICOLET 8700 spectrometer (Thermo Scientific, USA) using a single-bounce ATR accessory equipped with a Ge crystal at an angle of incidence of $45^{\circ}$. The spectral resolution and the number of scans were $2 \mathrm{~cm}^{-1}$ and 64 , respectively, for each measurement. A pressure clamp was used to achieve sufficient spectral quality, which depends on a good contact between the crystal and investigated sample. The acquired spectra were analyzed using the OMNIC TM $\mathrm{v} 8.1$ spectroscopic software.

$\mathrm{X}$-ray photoelectron spectroscopy (XPS) was used to quantify the chemical composition of LDPE samples. For this purpose, a TFA XPS Physical Electronics (USA) XPS instrument was used. The pressure in the chamber was approximately $6 \cdot 10^{-8} \mathrm{~Pa}$. The LDPE samples were irradiated with $\mathrm{X}$-rays over a $400 \mu \mathrm{m}$ spot area using monochromatic $\mathrm{Al} \mathrm{K}_{\alpha 1,2}$ radiation at $1486.6 \mathrm{eV}$. The photoelectrons were detected with a hemispherical analyzer placed at an angle of $45^{\circ}$ with respect to the normal to the surface of the LDPE samples. Each survey-scan spectrum was collected at a pass energy of $187.85 \mathrm{eV}$ and at an energy step of $0.4 \mathrm{eV}$. An electron gun was used for surface neutralization. The concentration of individual elements was determined using the MultiPak v7.3.1 software (Physical Electronics).

\subsection{Antibacterial efficacy tests}

The antibacterial effect of treated LDPE samples was determined against the S. aureus (CCM 4516) bacterial strain via measurement of an inhibition zone diameter on agar. Nutrient agar No. 2 M1269 
- $500 \mathrm{~g}$ (HiMedia Laboratories PII, Ltd.) was used in this study. Circle-shaped LDPE samples (diameter $\approx 8 \mathrm{~mm}$ ) were washed with ethanol, dried and placed on an agar plate inoculated with the bacterial suspension $($ volume $=100 \mu \mathrm{L}$, concentration $=$ $10^{7}$ units $\mathrm{mL}^{-1}$ ). The samples were incubated for $24 \mathrm{~h}$ at $37^{\circ} \mathrm{C}$. After $24 \mathrm{~h}$, the diameters of the inhibition zone were measured in 5 directions to obtain average values; the test was repeated three times. The inhibition zone area was calculated as the area of annulus between the inhibition zone borders and the LDPE samples.

\section{Results and discussion}

\subsection{Surface wettability}

The ability of liquid to remain in contact with the solid surface is expressed by the surface's wettability. The wettability belongs among a very important factor supporting the antibacterial behavior. Hydrophilic brush layer can be responsible for attraction of water and form a repellent layer on the surface. There is steric hindrance for proteins or microorganisms to adsorb to the surface and the adsorption is reduced by several orders of magnitude [26]. For the above mentioned reason, the wettability measurement has very high importance for a characterization of the antibacterial surfaces. The wettability denotes the angle between the liquid drop and the solid surface. The contact-angle measurements were used to determine the wettability. The contact angle of several testing liquids was used to evaluate the surface free energy. The surface free energy indicates reversible work done for a unit surface area creation. The parameters related to wettability, such as the contact angle of testing liquids, the total surface free energy $\left(\gamma^{\text {tot }}\right)$ and the dispersive $\left(\gamma^{\mathrm{d}}\right)$ and polar $\left(\gamma^{\mathrm{p}}\right)$ components of the free energy and $G Y$ are summarized in Table 1.

Given the hydrophobic and chemically inert nature of the surface of LDPE, the contact angles of the investigated testing liquids were relatively high. A significant decrease in contact angles was observed after plasma treatment of LDPE samples because of the incorporation of characteristic polar functional groups such as $\mathrm{C}=\mathrm{O},-\mathrm{OH}, \mathrm{COOH}, \mathrm{COO}-$, and $\mathrm{C}-\mathrm{O}-\mathrm{C}$ into the surface. The next step, which consisted of the radical graft polymerization of AA, resulted in a further decrease in the contact angles due to the polar character of PAA. The presence of bronopol and benzalkonium chloride also led to decreased contact angles in comparison to those of untreated LDPE. On the basis of contact angle measurements, we calculated the values of the surface energy and its components. Untreated LPDE is characterized by a very low surface energy, consistent with its hydrophobic character. The plasma treatment of LDPE resulted in an increase in the surface free energy due to the increased surface polarity. LDPE modified with polar PAA, bronopol and benzalkonium chloride exhibited very high surface free energy values. The graft yield of LDPE samples modified with antibacterial agent increased in compare with LDPE modified by PAA as result of introduction of benzalkonium chloride and bronopol on PAA brushes. The value of $G Y$ in case of benzalkonium chloride introduction was higher than in case of bronopol probably because of higher immobilization efficiency of benzalkonium chloride on PAA brushes.

\subsection{Adhesive properties}

The wettability closely relates to adhesion, which can be characterized by peel-test measurements. The results obtained from these measurements are presented in Figure 5. The adhesion of polymers correlates to their surface energy and to their roughness. Increased wettability of the LDPE surfaces results in increased adhesion and vice versa. Moreover, the increase in surface roughness is correlated with the adhesion increase due to the increase of surface con-

Table 1. Surface properties of LDPE modified via a multistep process $\left(\theta-\right.$ contact angle, $\gamma^{\text {tot }}-$ total surface free energy, $\gamma^{\mathrm{d}}$, $\gamma^{\mathrm{p}}$-dispersive and polar components of the surface free energy, respectively; $G Y$ - graft yield)

\begin{tabular}{|c|c|c|c|c|c|c|c|c|c|}
\hline LDPE sample & $\begin{array}{l}\theta_{w} \\
{\left[{ }^{\circ}\right]}\end{array}$ & $\begin{array}{l}\theta_{\mathrm{e}} \\
{\left[{ }^{\circ}\right]}\end{array}$ & $\begin{array}{l}\boldsymbol{\theta}_{\mathrm{g}} \\
{\left[{ }^{\circ}\right]}\end{array}$ & $\begin{array}{l}\boldsymbol{\theta}_{\mathbf{d}} \\
{\left[{ }^{\circ}\right]}\end{array}$ & $\begin{array}{l}\boldsymbol{\theta}_{\mathrm{f}} \\
{\left[{ }^{\circ}\right]}\end{array}$ & $\begin{array}{c}\gamma^{\text {tot }} \\
{\left[\mathrm{mJ} / \mathrm{m}^{2}\right]}\end{array}$ & $\begin{array}{c}\gamma^{\mathrm{d}} \\
{\left[\mathrm{mJ} / \mathrm{m}^{2}\right]}\end{array}$ & $\begin{array}{c}\gamma^{\mathrm{p}} \\
{\left[\mathrm{mJ} / \mathrm{m}^{2}\right]}\end{array}$ & $\begin{array}{l}\text { GY } \\
{[\%]}\end{array}$ \\
\hline Untreated & $99.2( \pm 0.6)$ & $70.9( \pm 1.2)$ & $85.3( \pm 0.9)$ & $48.4( \pm 1.2)$ & $80.7( \pm 0.9)$ & 31.7 & 31.5 & 0.2 & \\
\hline Plasma-treated (A) & $77.5( \pm 1.1)$ & $51.0( \pm 2.8)$ & $67.1( \pm 2.8)$ & $36.0( \pm 1.2)$ & $52.8( \pm 1.5)$ & 42.6 & 41.4 & 1.1 & 0.0 \\
\hline $\mathrm{A}+$ PAA grafted (B) & $66.9( \pm 0.7)$ & $32.1( \pm 2.4)$ & $57.2( \pm 2.7)$ & $32.5( \pm 1.6)$ & $37.0( \pm 2.0)$ & 48.1 & 43.7 & 4.5 & 0.5 \\
\hline $\mathrm{B}+$ benzalkonium chloride coated & $70.7( \pm 0.4)$ & $52.9( \pm 2.2)$ & $58.9( \pm 2.0)$ & $40.3( \pm 0.8)$ & $37.2( \pm 2.7)$ & 43.1 & 36.4 & 6.7 & 3.4 \\
\hline B + bronopol coated & $73.1( \pm 1.2)$ & $31.8( \pm 3.0)$ & $65.6( \pm 2.5)$ & $37.5( \pm 1.0)$ & $36.8( \pm 2.5)$ & 45.6 & 40.4 & 5.2 & 0.9 \\
\hline
\end{tabular}




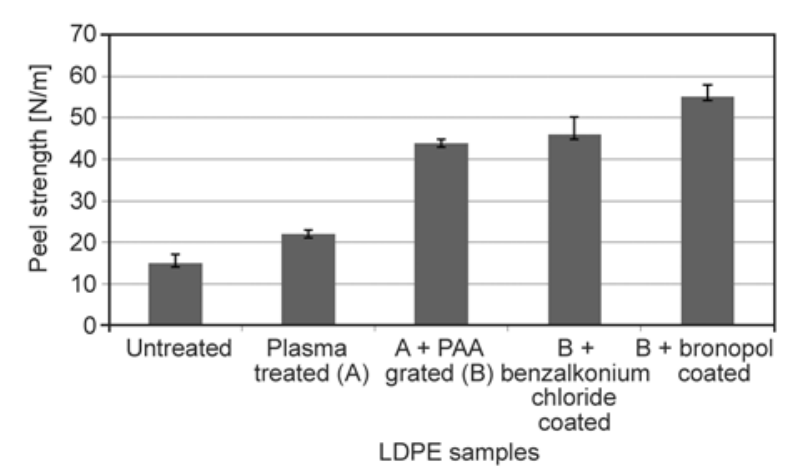

Figure 5. Changes in peel strength after each particular step of the LDPE antibacterial treatment

tact area. Adhesion is a complex phenomenon consisting of several chemical and physicochemical factors, as demonstrated in aforementioned results. A relatively low value of peel strength for the untreated LDPE was observed as result of the hydrophobic and chemically inert nature of the polymer. Moreover, the roughness of untreated LDPE was relatively low. The plasma treatment led to a more than twofold increase in the peel strength, caused by changes in the polarity and surface morphology. The grafting of PAA onto LDPE resulted in increased peel strength. The peel strength also increased in the cases of benzalkonium-chloride- and bronopol-coated LDPE samples.

\subsection{Surface morphology}

Changes in the surface roughness of untreated and antibacterial-treated LDPE were determined by AFM (Figure 6). Details related to the increase or decrease of the surface roughness were compared via the profile roughness parameter $\left(R_{\mathrm{a}}\right)$. The $R_{\mathrm{a}}$ value represents the average height of the irregularities in a perpendicular direction toward the sample surface, and, in the case of untreated LDPE, was being $38.1 \mathrm{~nm}$ in $10 \mu \mathrm{m}$ of scan area (Figure 6a) as a consequence of the procedure used to prepare the foils. The plasma treatment led to a decrease in the roughness, as indicated by an decrease in the $R_{\mathrm{a}}$ value to $21.9 \mathrm{~nm}$ (Figure 6b) as a result of surface changes induced by the functionalization and ablation processes. In the case of the AA radical graft polymerization step, the $R_{\mathrm{a}}$ value increased compared to that of the plasma-treated LDPE surface because PAA brushes were created on the LDPE surface (Figure 6c). The agglomerates created from bronopol resulted in an increase of the surface roughness; therefore, the $R_{\mathrm{a}}$ values bronopol reached $37.2 \mathrm{~nm}$
(Figure 6d) while benzalkonium chloride formed more uniform layer and $R_{\mathrm{a}}$ was $29.2 \mathrm{~nm}$ (Figure 6e).

\subsection{Surface chemistry analysis}

Semi-quantitative information about chemical changes in the near-surface region was obtained from FTIR-ATR measurements. The spectrum of pristine LDPE is characterized by bands (Figure 7, curve a) associated with $-\mathrm{CH}_{2}-$ stretching (2848 and $2915 \mathrm{~cm}^{-1}$ ), asymmetric $\mathrm{C}-\mathrm{H}$ and $-\mathrm{CH}_{3}$ bending in plane (1460 and $1470 \mathrm{~cm}^{-1}$, respectively), symmetric $\mathrm{C}-\mathrm{H}$ bending in plane $\left(1377 \mathrm{~cm}^{-1}\right)$, and $\mathrm{C}-\mathrm{H}\left(-\mathrm{CH}_{2}-\right)_{\mathrm{n} \geq 6}$ rocking (doublet with maxima at 720 and $731 \mathrm{~cm}^{-1}$ ) [27]. The plasma surface treatment of LDPE resulted in significant spectral changes (Figure 7, curve b), which provided clear evidence of the incorporation of certain oxygencontaining groups, i.e., -OH (region 3700$3080 \mathrm{~cm}^{-1}$ ) and/or other oxygen-containing products at the surface of the polymer (region 1845-1510, $1280,1126,1150 \mathrm{~cm}^{-1}$, acid, ketone, aldehyde) [28]. The modification of LDPE by PAA grafting and by subsequent treatment with benzalkonium chloride and bronopol led to additional spectral changes. Characteristic peaks of PAA grafted onto LDPE were observed, specifically, the most intense peak at $1712 \mathrm{~cm}^{-1}$ (carbonyl band, $\mathrm{C}=\mathrm{O}$ stretching) and some unresolved peaks in the fingerprint region (1300-1100 $\mathrm{cm}^{-1}, \mathrm{C}-\mathrm{O}$ stretching and $\mathrm{CH}_{2}$ bending), as shown in Figure 7, curve c. The presence of benzalkonium chloride was confirmed by the presence of the absorbance band at $702 \mathrm{~cm}^{-1}$, which is attributed to $\mathrm{C}-\mathrm{Cl}$ vibrations (Figure 7 , curve d). The spectrum of LDPE treated with bronopol shows characteristic peaks at $3300 \mathrm{~cm}^{-1}(-\mathrm{OH}), 1557$, $1340 \mathrm{~cm}^{-1}\left(-\mathrm{NO}_{2}\right)$ and $852 \mathrm{~cm}^{-1}(\mathrm{C}-\mathrm{Br})$ resulting from the incorporation of bronopol into the LDPE surface (Figure 7, curve e).

XPS was used to quantify the characteristic presence of typical chemical elements after each step of the antibacterial treatment. The surface composition of the LDPE samples, which is shown in Table 2, was measured at two different spots, allowing the average surface composition to be calculated.

The high resolution $\mathrm{C} 1 \mathrm{~s}$ spectra of all samples are shown in Figure 8. In the spectrum of untreated LDPE, only a C1s peak was present (Figure 8a), which is attributed to $\mathrm{C}-\mathrm{C} / \mathrm{C}-\mathrm{H}$ bonds. The greatest amount of oxygen and also nitrogen functional 

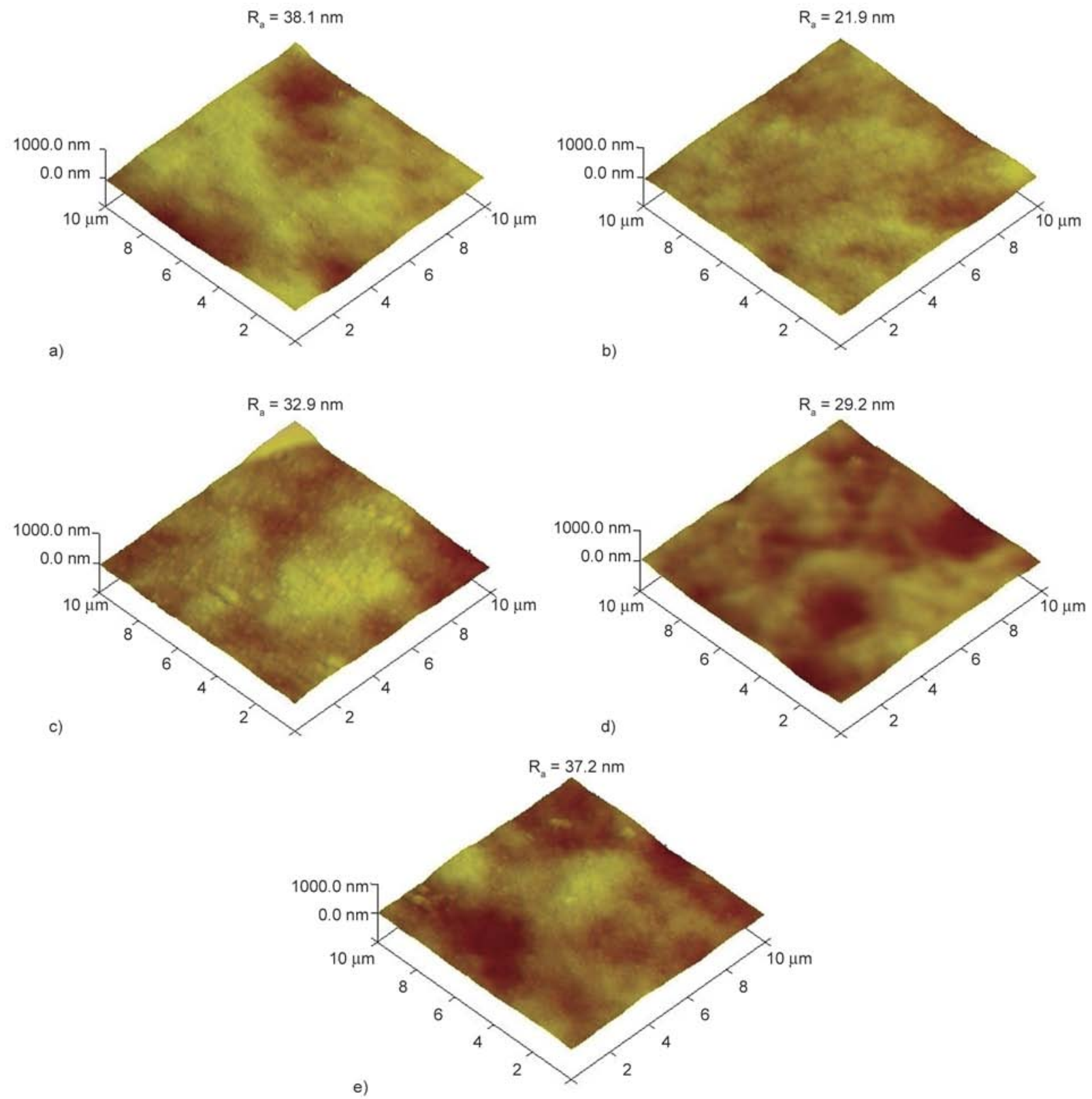

Figure 6. AFM images of LDPE sample surfaces and the samples' corresponding $R_{\mathrm{a}}$ values: a) untreated; $\mathrm{b}$ ) plasma treated (A); c) A + PAA grafted (B); d) B + benzalkonium chloride coated; and e) B + bronopol coated

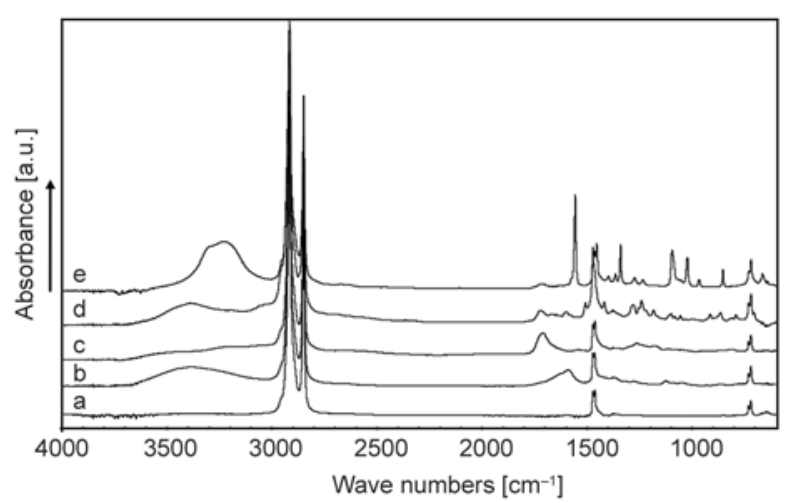

Figure 7. FTIR-ATR spectra of LDPE samples: a) untreated; b) plasma treated (A); c) A + PAA grafted (B); d) B + benzalkonium chloride coated; and e) $\mathrm{B}+$ bronopol coated groups (19.8 and 4.0 at $\%$, respectively) were observed in the plasma-treated LDPE sample. In this case, the carbon $\mathrm{C} 1 \mathrm{~s}$ peak represents $\mathrm{C}-\mathrm{C} / \mathrm{C}-\mathrm{H}$, $\mathrm{O}=\mathrm{C}-\mathrm{O}, \mathrm{C}=\mathrm{O}$, and $\mathrm{C}-\mathrm{O}$ groups (Figure $8 \mathrm{~b}$ ) and the nitrogen $\mathrm{N} 1 \mathrm{~s}$ peak is composed from different chemical bonds of nitrogen atoms such as $\mathrm{C}-\mathrm{N}, \mathrm{C}-\mathrm{NH}_{3}^{+}$, and $-\mathrm{ONO}_{2}$. The spectrum of the LDPE sample grafted with PAA showed mainly the presence of an O1s peak as a consequence of the incorporation of carboxyl groups (Figure 8c); however, this peak may also indicate the presence of other oxygen groups created during plasma treatment. These samples also contained some iron traces and sulfur 
Table 2. Surface composition of LDPE samples, as obtained from XPS measurements

\begin{tabular}{|c|c|c|c|c|c|c|c|}
\hline \multirow{2}{*}{ LDPE samples } & C1s & N1s & O1s & Cl2p & Si2p & S2p & Br3d \\
\hline & \multicolumn{7}{|c|}{ [at\%] } \\
\hline Untreated & 100.0 & 0 & 0 & & & & \\
\hline Plasma treated (A) & 76.3 & 4.0 & 19.8 & & & & \\
\hline A + PAA grafted (B) & 84.1 & 0 & 15.6 & & & 0.4 & \\
\hline $\mathrm{B}+$ benzalkonium chloride coated & 87.8 & 3.0 & 4.7 & 4.5 & & & \\
\hline $\mathrm{B}+$ bronopol coated & 91.5 & 0 & 7.1 & & 1.3 & & 0.1 \\
\hline
\end{tabular}
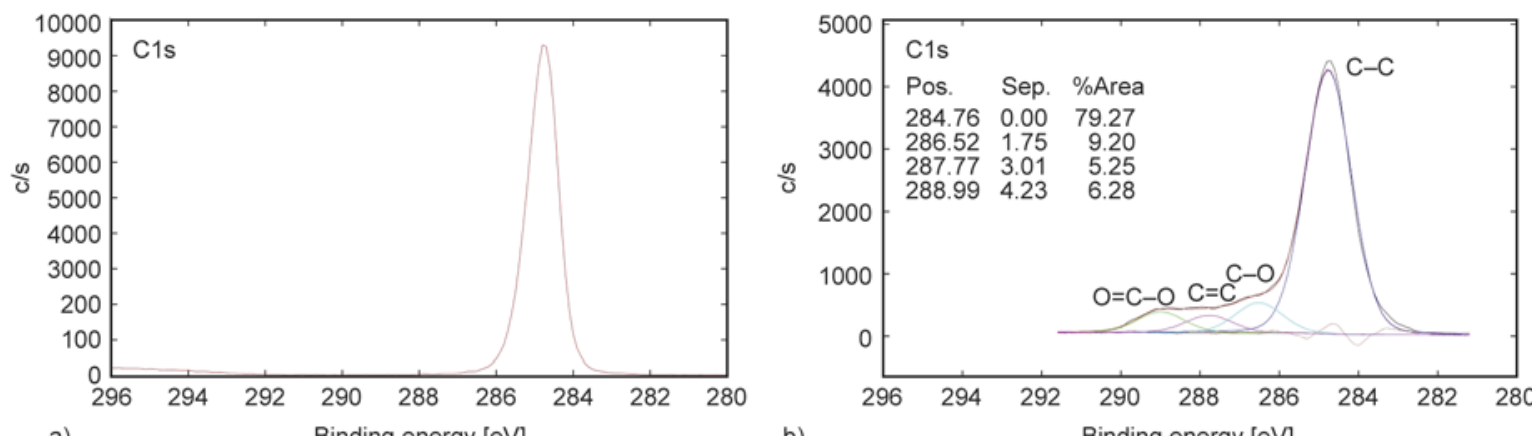

a)

b)
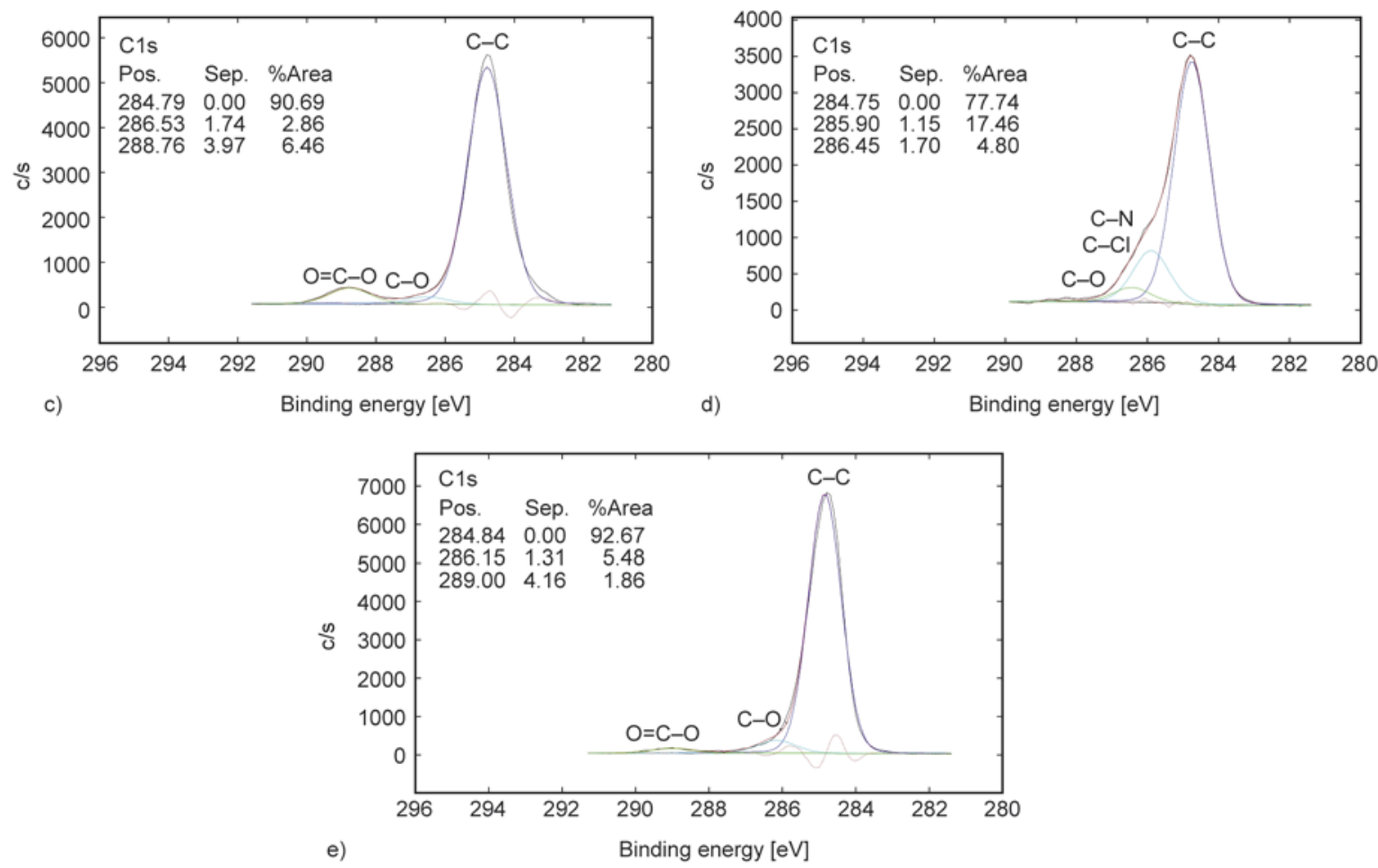

Figure 8. High resolution C1s spectra of LDPE samples: a) untreated; b) plasma treated (A); c) A + PAA grafted (B); d) B + benzalkonium chloride coated; and e) B + bronopol coated

impurities (approximately 0.4 at $\%$ ) which probably originated from dust or chemicals. XPS spectra of the benzalkonium chloride-coated LDPE sample revealed the presence of $\mathrm{N}$ and $\mathrm{Cl}$ (Figure 8d), which originate from the antibacterial agent. The presence of a small amount of $\operatorname{Br}(0.1$ at $\%)$ was detected in LDPE samples coated with bronopol, which is likely associated with the creation of the very thin bronopol layer.

\subsection{Antibacterial effect}

The antibacterial effect was determined by measurement of the inhibition zones. The results were averaged to obtain the one representative value when all three repeated measurements of the LDPE samples treated in the same manner exhibited some antibacterial activity. The measurements clearly indicated an antibacterial effect against $S$. aureus. In Figure 9, the inhibition zone values for each step 
of the antibacterial treatment are presented. Samples coated with bronopol or benzalkonium chloride were active against $S$. aureus, which confirmed presence of the antibacterial agent. The best results were obtained for LDPE samples coated with benzalkonium chloride. Moreover, the use of PAA as an active platform for the immobilization of antibacterial agents had a more pronounced effect on the antibacterial activity against $S$. aureus in comparison to, e.g., LDPE samples grafted by allylamine, reported elsewhere [29]. Antibacterial activity against E. coli was not sufficiently demonstrated.

\section{Conclusions}

This work was focused on the anchoring of selected antibacterial agents to the surface of LDPE through the use of low-temperature plasma and PAA grafting on pre-treated samples. Such treated LDPE samples exhibited changes in their surface and chemical composition. The immobilization of the antibacterial agents led to changes in the surface roughness of LDPE which was confirmed by the $R_{\mathrm{a}}$ value increase from $21.9 \mathrm{~nm}$ for the plasma treated LDPE to 29.2 and $37.2 \mathrm{~nm}$ for PE surfaces grafted with benzalkonium chloride and bronopol, respectively. Moreover, this antibacterial treatment significant affected the wettability and adhesion of LDPE as a consequence of the increase in roughness and hydrophilicity, as previously mentioned, whereas the $\gamma^{\text {tot }}$ of LDPE increased from 31.7 to $43.1 \mathrm{~mJ} / \mathrm{m}^{2}$ and $45.6 \mathrm{~mJ} / \mathrm{m}^{2}$ for LDPE surfaces covered with benzalkonium chloride and bronopol, respectively. The increase in hydrophilicity was caused by the introduction of polar functional groups present in the applied antibacterial agents, as was proven by FTIR-ATR and XPS measurements. The antibacterial effect of such

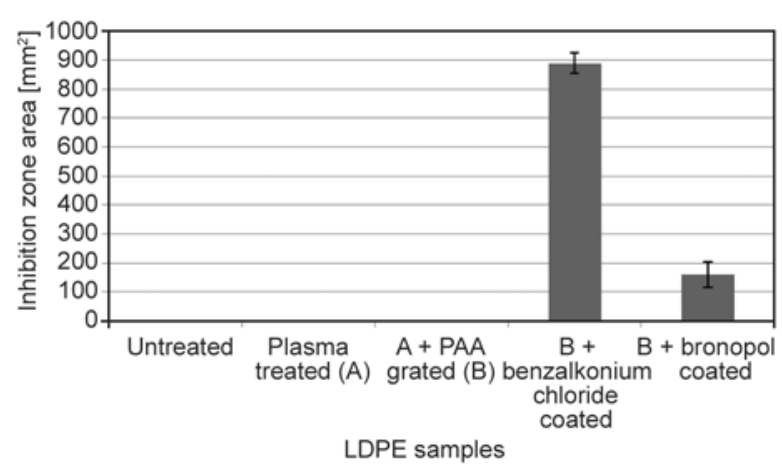

Figure 9. The inhibition zone area of LDPE samples after each step of the antibacterial treatment treated LDPE films was confirmed by measurements of the inhibition zone of $S$. aureus; the most pronounced antibacterial activity was exhibited by LDPE samples treated with plasma, grafted with PAA, and subsequently coated with benzalkonium chloride. The antibacterial effect against $E$. coli was not sufficiently proven.

\section{Acknowledgements}

Financial support by the Ministry of Education, Youth, and Sports of the Czech Republic (Grant CZ.1.05/2.1.00/ 03.0111), the Slovak Academy of Sciences (Grant VEGA 2/0151/12 and 2/0199/14), Grant Agency of the Czech Republic (Czech Science Foundation, project No. 1306700S and 13-08944S) are gratefully acknowledged. Author M. Mozetič would like to express his gratitude to the Slovenian Research Agency.

\section{References}

[1] Sanchis R., Fenollar O., García D., Sánchez L., Balart R.: Improved adhesion of LDPE films to polyolefin foams for automotive industry using low-pressure plasma. International Journal of Adhesion and Adhesives, 28, 445-451 (2008). DOI: $10.1016 /$ j.ijadhadh.2008.04.002

[2] Kuzuya M., Sawa T., Mouri M., Kondo S-I., Takai O.: Plasma technique for the fabrication of a durable functional surface on organic polymers. Surface and Coatings Technology, 169-170, 587-591 (2003). DOI: $10.1016 / \mathrm{S} 0257-8972(03) 00116-6$

[3] Zhang W., Chu P. K., Ji J., Zhang Y., Fu R. K. Y., Yan Q.: Antibacterial properties of plasma-modified and triclosan or bronopol coated polyethylene. Polymer, 47, 931-936 (2006).

DOI: $10.1016 /$ j.polymer.2005.12.009

[4] Costa F., Carvalho I. F., Montelaro R. C., Gomes P., Martins M. C.: Covalent immobilization of antimicrobial peptides (AMPs) onto biomaterial surfaces. Acta Biomaterialia, 7, 1431-1440 (2011).

DOI: $10.1016 /$ j.actbio.2010.11.005

[5] Newton A. P. N., Cadena S. M. S. C., Rocha M. E. M., Carnieri E. G. S., de Oliveira M. B. M.: Effect of triclosan (TRN) on energy-linked functions of rat liver mitochondria. Toxicology Letters, 160, 49-59 (2005). DOI: $10.1016 /$ j.toxlet.2005.06.004

[6] Suzuki M., Kishida A., Iwata H., Ikada Y.: Graft copolymerization of acrylamide onto a polyethylene surface pretreated with glow discharge. Macromolecules, 19, 1804-1808 (1986). DOI: $10.1021 / \mathrm{ma} 00161 \mathrm{a} 005$

[7] Johnsen K., Kirkhorn S., Olasen K., Redford K., Stori A.: Modification of polyolefin surfaces by plasmainduced grafting. Journal of Applied Polymer Science, 59, 1651-1657 (1996). DOI: $10.1002 /($ SICI)1097-4628(19960307)59:10<1651 ::AID-APP17>3.0.CO;2-Z 
[8] Asadinezhad A., Novák I., Lehocký M., Sedlařík V., Vesel A., Junkar I., Sáha P., Chodák I.: A physicochemical approach to render antibacterial surfaces on plasmatreated medical-grade PVC: Irgasan coating. Plasma Processes and Polymers, 7, 504-514 (2010). DOI: 10.1002/ppap.200900132

[9] Vesel A., Mozetic M.: Surface modification and ageing of PMMA polymer by oxygen plasma treatment. Vacuum, 86, 634-637 (2012). DOI: $10.1016 /$ j.vacuum.2011.07.005

[10] Junkar I., Vesel A., Cvelbar U., Mozetič M., Strnad S.: Influence of oxygen and nitrogen plasma treatment on polyethylene terephthalate (PET) polymers. Vacuum, 84, 83-85 (2010).

DOI: $10.1016 /$ j.vacuum.2009.04.011

[11] Vesel A., Mozetic M.: Surface functionalization of organic materials by weakly ionized highly dissociated oxygen plasma. Journal of Physics: Conference Series, 162, 012015/1-012015/20 (2009). DOI: $10.1088 / 1742-6596 / 162 / 1 / 012015$

[12] Wei Q. F.: Surface characterization of plasma-treated polypropylene fibers. Materials Characterization, 52, 231-235 (2004).

DOI: $10.1016 /$ j.matchar.2004.05.003

[13] Lloyd G., Friedman G., Jafri S., Schultz G., Fridman A., Harding K.: Gas plasma: Medical uses and developments in wound care. Plasma Processes and Polymers, 7, 194-211 (2010). DOI: 10.1002/ppap.200900097

[14] Slepička P., Slepičková Kasálková N., Stránská E., Bačáková L., Švorčík V.: Surface characterization of plasma treated polymers for applications as biocompatible carriers. Express Polymer Letters, 7, 535-545 (2013).

DOI: $10.3144 /$ expresspolymlett.2013.50

[15] Černák M., Černáková L., Hudec I., Kováčik D., Zahoranová A.: Diffuse coplanar surface barrier discharge and its applications for in-line processing of lowadded-value materials. The European Physical Journal Applied Physics, 47, 22806/1-22806/6 (2009). DOI: $10.1051 /$ epjap/2009131

[16] Šimor M., Ráhel' J., Vojtek P., Černák M., Brablec A.: Atmospheric-pressure diffuse coplanar surface discharge for surface treatments. Applied Physics Letters, 81, 2716-2718 (2002).

DOI: $10.1063 / 1.1513185$

[17] Černák M., Ráhel’ J., Kováčik D., Šimor M., Brablec A., Slavíček P.: Generation of thin surface plasma layers for atmospheric-pressure surface treatments. Contributions to Plasma Physics, 44, 492-495 (2004). DOI: $10.1002 /$ ctpp.200410069

[18] Saxena S., Ray A. R., Mindemart J., Hilborn J., Gupta B.: Plasma-induced graft polymerization of acrylic acid onto poly(propylene) monofilament: Characterization. Plasma Processes and Polymers, 7, 610-618 (2010). DOI: $\underline{10.1002 / \text { ppap. } 200900165}$
[19] Zhao B., Brittain W. J.: Polymer brushes: Surfaceimmobilized macromolecules. Progress in Polymer Science, 25, 677-710 (2000).

DOI: $10.1016 / \mathrm{S} 0079-6700(00) 00012-5$

[20] Shi Z., Neoh K. G., Zhong S. P., Yung L. Y. L., Kang E. Y., Wang W.: In vitro antibacterial and cytotoxicity assay of multilayered polyelectrolyte-functionalized stainless steel. Journal of Biomedical Materials Research Part A, 76, 826-834 (2006).

DOI: $10.1002 / \mathrm{jbm} . \mathrm{a} .30597$

[21] Wang H., Provan G. J., Helliwell K.: Determination of bronopol and its degradation products by HPLC. Journal of Pharmaceutical and Biomedical Analysis, 29, 387-392 (2002).

DOI: $10.1016 / \mathrm{S} 0731-7085(02) 00078-\mathrm{X}$

[22] Tiller J. C.: Coatings for prevention or deactivation of biological contamination. in 'Developments in surface contamination and cleaning' (eds.: Kohli R., Mittal K. L.) Elsevier, Norwich, Vol 1, 1013-1065 (2007).

[23] Bílek F., Sulovská K., Lehocký M., Sáha P., Humpolíček P., Mozetič M., Junkar I.: Preparation of active antibacterial LDPE surface through multistep physicochemical approach II: Graft type effect on antibacterial properties. Colloids and Surfaces B: Biointerfaces, 102, 842-848 (2013).

DOI: $10.1016 /$ j.colsurfb.2012.08.026

[24] Salimi A., Mirabedini S. M., Atai M., Mohseni M., Naimi-Jamal M. R.: Correlating the adhesion of an acrylic coating to the physico-mechanical behavior of a polypropylene substrate. International Journal of Adhesion and Adhesives, 31, 220-225 (2011).

DOI: 10.1016/j.ijadhadh.2011.01.003

[25] Işiklan N., Kurşun F., İnal M.: Graft copolymerization of itaconic acid onto sodium alginate using benzoyl peroxide. Carbohydrate Polymers, 79, 665-672 (2010). DOI: 10.1016/j.carbpol.2009.09.021

[26] Knetsch M. L. W., Koole L. H.: New strategies in the development of antimicrobial coatings: The example of increasing usage of silver and silver nanoparticles. Polymers, 3, 340-366 (2011).

DOI: $10.3390 /$ polym3010340

[27] Asensio R. Ch., Moya M. S. A., de la Roja J. M., Gómez M.: Analytical characterization of polymers used in conservation and restoration by ATR-FTIR spectroscopy. Analytical and Bioanalytical Chemistry, 395, 2081-2096 (2009).

DOI: $10.1007 / \mathrm{s} 00216-009-3201-2$

[28] Sellin N., de C. Campos J. S.: Surface composition analysis of PP films treated by corona discharge. Materials Research, 6, 163-166 (2003). DOI: $10.1590 /$ S1516-14392003000200009

[29] Bílek F., Křížová T., Lehocký M.: Preparation of active antibacterial LDPE surface through multistep physicochemical approach: I. Allylamine grafting, attachment of antibacterial agent and antibacterial activity assessment. Colloids and Surfaces B: Biointerfaces, 88, 440447 (2011).

DOI: $10.1016 /$ j.colsurfb.2011.07.027 